\title{
Application of the nanoindentation method in assessing of properties of cement composites modified with silica-magnetite nanostructures
}

\author{
Elzbieta Horszczaruk $^{1,}{ }^{*}$, Roman Jedrzejewski ${ }^{2}$, Jolanta Baranowska $^{2}$, and Ewa Mijowska ${ }^{3}$ \\ ${ }^{1}$ Faculty of Civil Engineering and Architecture, West Pomeranian University of Technology, \\ Szczecin, Al. Piastow 50, 70-311 Szczecin, Poland \\ ${ }^{2}$ Faculty of Mechanical Engineering and Mechatronics, West Pomeranian University of Technology, \\ Szczecin, Al. Piastow 19, 70-310 Szczecin, Poland \\ ${ }^{3}$ Faculty of Chemical Technology and Engineering, West Pomeranian University of Technology, \\ Szczecin, Al. Piastow 45, 70-311 Szczecin, Poland
}

\begin{abstract}
The results of investigation of the cement composites modified with $5 \%$ of silica-magnetite nanostructures of the core-shell type are presented in the paper. The nanoindentation method employing three-sided pyramidal Berkovich indenter was used in the research. The mechanical properties and microstructure of the modified cement composites were evaluated on the basis of the values of hardness and indentation modulus measured inside the cement matrix and in the aggregate-paste interfacial zone. The results were compared with those obtained for the reference composites without nanostructures. The positive influence of the presence of silica-magnetite nanoparticles on the tested properties was found out.
\end{abstract}

\section{Introduction}

Recently, new testing techniques have been efficiently used for the control of properties and durability of the cement-based materials, particularly the cement composites modified with addition of various types of nanoparticles. In the cement composites, like concrete or mortar, the weakest place is still the interfacial transition zone between the cement matrix and the aggregate or other filler, as well as between the components of the matrix itself. The transition zone affects significantly the macro-properties of the cement concretes, like strength, modulus of elasticity or crack propagation. One of the reason for use of nanomaterials in the cement composites is strengthening of the transition zone. The nanoindentation method is increasingly used in testing of nanoproperties of composites modified in this way [1-6].

The basic difficulty in verification of the values of elasticity modulus and hardness of the cement composites, obtained using the nanoindentation method, is the proper selection of the scale of lattice, pressing force and type of indenter. The authors of [7] have observed the high variability of the obtained results of measurements of the elasticity modulus for the phases of the cement paste. This variability is a result of use of the different loadings and indenters

\footnotetext{
*Corresponding author: elzbieta.horszczaruk@zut.edu.pl
} 
as well as the variable assumptions regarding to the scale of lattice, often maladjusted to the structure of the tested composite.

The results of investigation of the mechanical properties of cement composites modified with admixture of silica-magnetite nanostructures are presented in the paper. The basic goal in the use of such nanostructures was, apart from improvement of shielding properties of the composites on gamma-rays, achieving of better resistance of the composite on high temperature [8]. Therefore, the subject of the detailed investigation was the characteristic of the aggregate-paste contact zone. For this aim, the nanoindentation method was used.

\section{Materials and methods}

\subsection{Materials and specimens preparation}

The cement composites were made using portland cement CEM I 42,5R; the composition of the cement is presented in the Table 1. Two types of the cement pastes were prepared with water to cement ratio $\mathrm{w} / \mathrm{c}=0.5$. The first paste $(\mathrm{R})$ was the reference one and contained no nanoparticles, while the second paste (N5) contained 5\% (refering to the cement mass) of the silica-magnetite nanostructures of the core-shell type (Table 2).

$\mathrm{The} \mathrm{Fe}_{3} \mathrm{O}_{4}$ particles used as a core for the nanostructures were purchased from Sigma Aldrich (637106). The nanomagnetite structures were in the size of $50-100 \mathrm{~nm}$. In order to create a silica shell on the surface of the nanomagnetite $\left(\mathrm{Fe}_{3} \mathrm{O}_{4} / \mathrm{nSiO}_{2}\right), 2 \mathrm{~g}$ of $\mathrm{Fe}_{3} \mathrm{O}_{4}$ were dispersed in $200.0 \mathrm{~mL}$ of ethanol $(\mathrm{EtOH})$, mixed together with $2.5 \mathrm{~mL}$ of ammonia solution $\left(\mathrm{NH}_{3} \cdot \mathrm{H}_{2} \mathrm{O}\right)$. Next, $1.5 \mathrm{~mL}$ of tetraethyl orthosilicate (TEOS) was added. The resultant mixture was stirred for $18 \mathrm{~h}$ at room temperature. The particles were separated with a magnet, washed with distilled water and air-dried. Detailed description of synthesis process is reported in [9]. Transmission electron micrograph (TEM) of the pristine $\left(\mathrm{Fe}_{3} \mathrm{O}_{4}\right)$ and silica coated $\left(\mathrm{Fe}_{3} \mathrm{O}_{4} / \mathrm{nSiO}_{2}\right)$ magnetite nanoparticles are shown in the Figure 1.

Table 1. Chemical composition of the portland cement (wt.\%).

\begin{tabular}{|c|c|c|c|c|c|c|c|c|c|}
\hline Material & $\mathbf{S i O}_{2}$ & $\mathbf{A l}_{2} \mathbf{O}_{\mathbf{3}}$ & $\mathbf{F e}_{2} \mathbf{O}_{\mathbf{3}}$ & $\mathbf{C a O}$ & $\mathbf{M g O}$ & $\mathbf{S O}_{\mathbf{3}}$ & $\mathbf{N a}_{2} \mathbf{O}$ & $\mathbf{K}_{2} \mathbf{O}$ & $\mathbf{C r}^{-}$ \\
\hline CEM I 42.5R & 20.2 & 5.0 & 2.6 & 64.1 & 1.58 & 2.5 & 0.2 & 0.7 & 0.06 \\
\hline
\end{tabular}

Table 2. Proportions of the cement pastes.

\begin{tabular}{|c|c|c|c|}
\hline Sample designation & Cement & Nano-particles & Water \\
\hline R & 1.0 & 0 & 0.5 \\
\hline N5 & 1.0 & 0.05 & 0.5 \\
\hline
\end{tabular}

The specimens for investigation of the aggregate-paste contact zone were prepared in the steel cubic moulds with side length of $20 \mathrm{~mm}$. For this aim, the grain of the river gravel with dimensions 10-12 mm was placed on the bottom of the mould and flooded with the cement paste. The specimens were demoulded after 24 hours and then stored for 28 days in the container filled with water at the temperature $20 \pm 2^{\circ} \mathrm{C}$. After removing from water, the specimens were cut in half, and the surfaces with the cut aggregate were additionally treated for nanoindentation testing of the interfacial zone. The method of surface treatment is described in 2.2. 

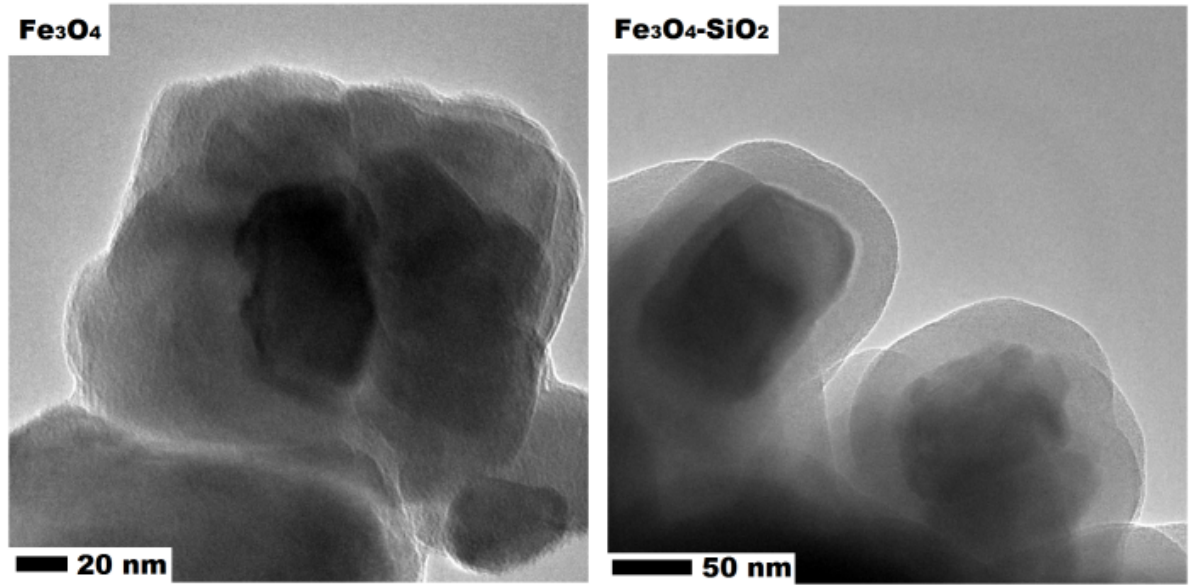

Fig. 1. TEM images of $\mathrm{Fe}_{3} \mathrm{O}_{4}$ and $\mathrm{Fe}_{3} \mathrm{O}_{4} / \mathrm{nSiO}_{2}$ nanoparticles.

\subsection{Nanoindentation test}

The surface of the concrete specimens needs special preparation for nanoindentation testing. For ensuring of the flat-parallelism of the surfaces, the specimens were fixed to the handle using thermal glue of the crystalbond type. Every specimen was polished using the sandpapers (the grain size: $200,400,800,1000,1200,1500,2000$, in sequence) in water for $\max .5 \mathrm{~min}$. Then the specimens were polished using the cloths with diamond suspensions with the grain size 9,6 and $3 \mu \mathrm{m}$, in sequence. Finally, the specimens were polished on the diamond films with the grain size 1 and $0.5 \mu \mathrm{m}$ (in ethanol, for 10-15 min.). After polishing the specimens were washed in the ultrasonic washer with ethanol for $5 \mathrm{~min}$.

The hardness and indentation modulus were measured by nanoindentation method, using Nanoindenter XP produced by Agilent Place and three-sided pyramidal Berkovich indenter. At least 15 series, each with 8 imprints in the row, was performed for every specimen. The first imprint was placed about 10-20 $\mu \mathrm{m}$ from the edge of the aggregate (Fig. 2). The distance between the imprints was about $20 \mu \mathrm{m}$.

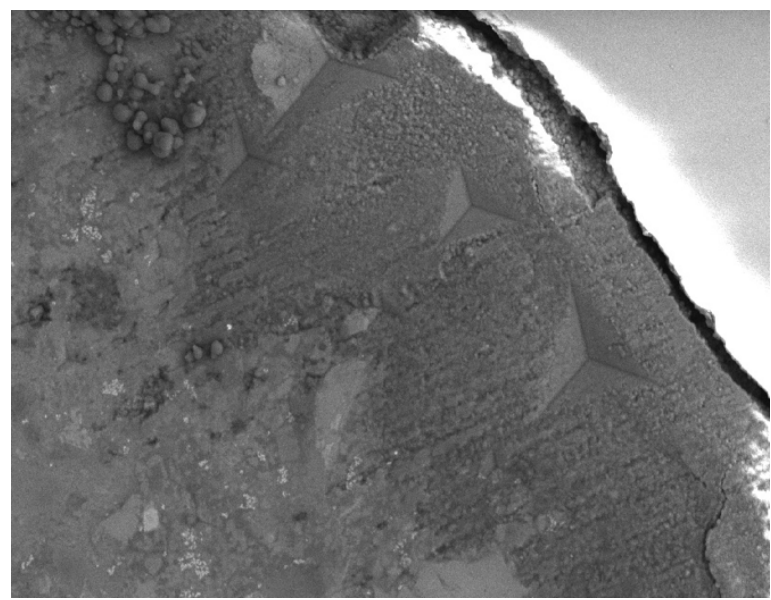

Fig. 2. SEM image of the imprints during the nanoindentation test. 


\section{Results and discussion}

The obtained results of nanoindentation tests were selected according to the measurement correctness. The values of hardness, $\mathrm{H}$, and indentation modulus, $\mathrm{M}$, were determined and statistically analyzed. The final results of hardness and modulus measurements have been averaged within the range of the values determined at the same distance from the aggregate. The mean values for the tested specimens are presented in the Table 3.

Table 3. The mean values of hardness and indentation modulus determined using nanoindentation method.

\begin{tabular}{|c|c|c|c|}
\hline \multicolumn{2}{|c|}{ Area } & R & N5 \\
\hline \multirow{2}{*}{$\begin{array}{c}\text { Interfacial } \\
\text { zone }\end{array}$} & $\mathrm{H}[\mathrm{GPa}]$ & 0.52 & 0.59 \\
\cline { 2 - 4 } & $\mathrm{M}[\mathrm{GPa}]$ & 14.21 & 17.72 \\
\hline \multirow{2}{*}{$\begin{array}{c}\text { Cement } \\
\text { matrix }\end{array}$} & $\mathrm{H}[\mathrm{GPa}]$ & 0.57 & 0.74 \\
\cline { 2 - 4 } & $\mathrm{M}[\mathrm{GPa}]$ & 17.53 & 24.11 \\
\hline
\end{tabular}

In the composite with nanoparticles admixture (N5), the hardness in the aggregate-paste contact zone was by $13 \%$ higher and inside the cement matrix by $29 \%$ higher than in the reference material (R). Similarly, the indentation modulus in the N5 composite was by $22 \%$ higher in the transition zone and by $37 \%$ higher inside the cement matrix, as compared to the reference sample (Fig. 3).

It has been found on the basis of the test results analysis that the width of the transition zone in the composite with nanoparticles (N5) is, on average, half as much as in the reference composite (R). This has been confirmed by measurements of the transition zone width performed using scanning microscope (SEM analysis). This width in the sample N5 was 25$40 \mu \mathrm{m}$, while in the case of the reference sample it was $40-70 \mu \mathrm{m}$.

Analysis of the results of nanoindentation testing shows that the admixture of silicamagnetite nanoparticles improves the properties of the cement composites inside the cement matrix and causes a decrease of the width of the transition zone, improving its mechanical nanoproperties.

The nanoindentation method makes possible to carry out testing on the relatively small specimens, what is a big advantage of this method, taking into consideration the high cost of nanomodifiers as well as long and often complicated production process. However, in the case of cement composites, the method requires very careful preparation of the samples and large number of the measurements as well as application of statistical analysis for the interpretaion of the test results

The improvement of the mechanical properties in the transition zone of the composite N5 can be explained by the presence of a shell of nano- $\mathrm{SiO}_{2}$. As it was confirmed in a number of researches [10-16], application of nanosilica in the cement composites accelerates the hydration process, improving the strength and microstructure of the composite. Investigation by Y. Qing et al. [17] showed that the use of nano- $\mathrm{SiO}_{2}$ leads to improvement of the bond in the aggregate-paste contact zone. 

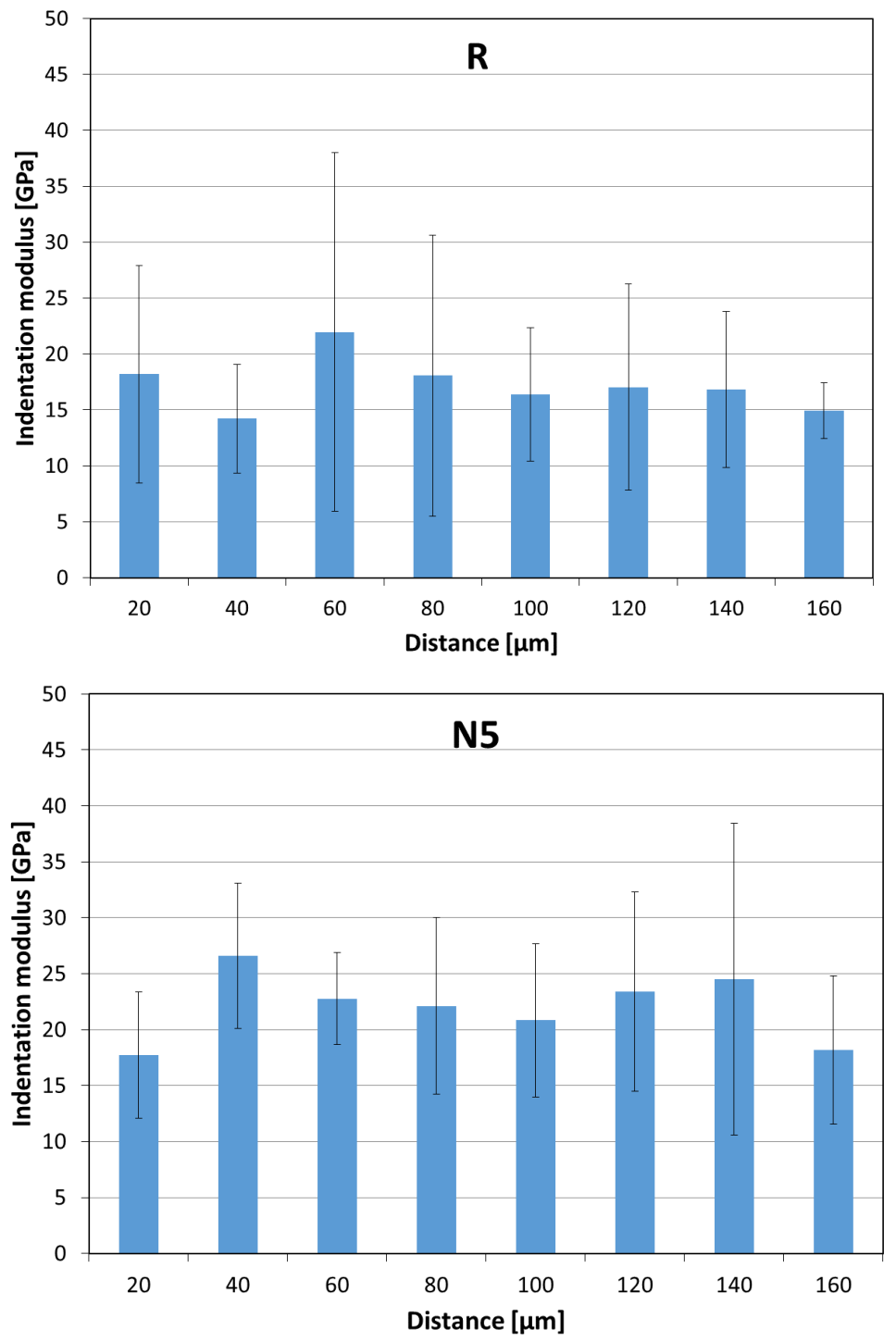

Fig. 3. Results of measurements of indentation modulus of the tested specimens in the transition zone.

\section{Summary}

Application of the nanoindentation method and SEM analysis made possible to describe the mechanical properties and structure of the aggregate-paste contact zone in the cement composites modified with silica-magnetite nanoparticles. The improvement of the mechanical properties of modified composite was observed both in the transition zone and inside the cement matrix itself, as compared to the reference composite. The reason for the decrease of the width of interfacial transition zone is application of the silica shell in the used nanostructures.

The nanoindentation method, when applied for the testing of cement composites, requires very careful preparation of the specimens. The roughness of the surfaces of the tested specimens should be as low as possible. 
This research was funded by the National Science Centre within the project No. 2014/13/B/ST8/03875 (OPUS 7).

\section{References}

1. G. Constantinides, F.-J. Ulm, K. Van Vliet, Mater. Struc. 36, 7 (2003)

2. M. Sebastiani, R. Moscatelli F. Ridi P. Baglioni, F. Carassiti, Mater. Des. 97, 9 (2016)

3. W.R.L. da Silva, J. Nemecek, P. Štemberk, Cem. Concr. Compos. 45, 12 (2014)

4. P. Trtik, B. Munch, P. Lura, Cem. Concr. Compos. 31, 10 (2009)

5. V. Zadeh, Ch. P. Bobko, Cem. Concr. Compos. 52, 9 (2014)

6. I. Zyganitidis,M. Stefanidou, N. Kalfagiannis, S. Logothetidis, Mater. Sci. Eng. B. 176, $5(2011)$

7. G. Constantinides G, F-J Ulm, J. Mech. Phys. Sol. 55, 26 (2007)

8. E. Horszczaruk, P. Sikora, K. Cendrowski, E. Mijowska, Constr. Build. Mater. 137, 12 (2017)

9. K. Cendrowski, P. Sikora, B. Zielinska, E. Horszczaruk, E. Mijowska, Appl. Surf. Sci. 407, 7 (2017)

10. B.W. Jo, C. H. Kim, Tae. G-h, J.-B. Park, Constr. Build. Mater. 21, 6 (2007)

11. E. Horszczaruk, E, Mijowska, K. Cendrowski, S. Mijowska, P. Sikora, Cement Lime Concrete 1, 9 (2013)

12. E. Horszczaruk, E, Mijowska, K. Cendrowski, S. Mijowska, P. Sikora, Constr. Build. Mater. 66 (2014)

13. K.L. Lin, W.C. Chang, D.F. Lin, H.L. Luo, M.C. Tsai , J. Environ. Manage. 88, 7 (2008)

14. M. Nili, A. Ehsani, Mater. Des.75, 10 (2015)

15. J. Schoepfer, A. Maji, ACI Spec. Publ. 267, 18 (2009)

16. P. Sikora, P. Lukowski, K. Cendrowski, E. Horszczaruk, E. Mijowska, Proced. Eng. 108, 7 (2015)

17. Y. Qing, Z. Zenan, K. Deyu, C. Rongshen, Constr. Build. Mater. 21, 7 (2007) 